\title{
Bortezomib Plus Melphalan-Induced Cardiomyopathy Presenting as Sinus Tachycardia and Systolic Heart Failure
}

\author{
Parth J. Sampat ${ }^{1}$, Sana Riaz ${ }^{1}$, Fidel Martinez ${ }^{2}$, Dana Aiello ${ }^{2}$ \\ 1. Internal Medicine, State University of New York Upstate Medical University, Syracuse, USA 2. Cardiology, State \\ University of New York Upstate Medical University, Syracuse, USA
}

Corresponding author: Parth J. Sampat, sampatp@upstate.edu

\begin{abstract}
Chemotherapy-induced cardiotoxicity is a known condition, however, bortezomib and melphalan do not typically cause cardiotoxicity. With the rise in the use of newer chemotherapeutic agents, it is important to identify and understand the cardiac implications of chemotherapeutic agents. We present a case of a 70year-old female with no known significant cardiac history presenting with partially reversible cardiomyopathy with initial presentation only being as sinus tachycardia.
\end{abstract}

Categories: Cardiology, Internal Medicine, Oncology

Keywords: cardiomyopathy, bortezomib, melphalan, sinus tachycardia, cardiotoxicity, systolic heart failure

\section{Introduction}

Multiple myeloma (MM) is a neoplastic proliferation of plasma cells [1]. The use of autologous stem cell transplantation and the availability of treatments such as immunomodulatory drugs and proteasome inhibitors have led to an increase in survival times [1]. Chemotherapy has been associated with cardiotoxicity in a variety of manners, which include hypertension, arrhythmias, heart failure, and so on [2]. Anthracyclines are widely considered as cardiotoxic which has limited its use [3]. Newer chemotherapy agents have lesser cardiovascular adverse events. Bortezomib is approved for the treatment of multiple myeloma and mantle cell lymphoma and is considered safer in terms of cardiovascular profile. Reports are suggesting that bortezomib is associated with cardiomyopathy and cardiotoxicity, however, is still considered rare [4-9]. The incidence of cardiotoxicity with the use of bortezomib-based regimens has been between $0-17.9 \%$ [7]. Melphalan is used for conditioning prior to hematopoietic stem cell transplant for the treatment of multiple myeloma [10]. The use of bortezomib and melphalan together has been found superior for the treatment of multiple myeloma [11]. Cardiomyopathy has been reported with high dose melphalan conditioning for autologous stem cell transplant with the incidence being approximately $1.6 \%$ with the use of melphalan for MM [12]. In biological experiments on rats, it has been found that bortezomib at higher concentrations increases the cytotoxic effects of melphalan on cardiac myocytes [13]. As the use of these medications has been increased in the past few years, understanding rare adverse effects are important, particularly cardiovascular toxicities. We present a case of multiple myeloma developing cardiotoxicity following the use of bortezomib and melphalan.

Received 07/08/2020 Review ended 07/19/2020 Published 07/31/2020

\section{(c) Copyright 2020}

Sampat et al. This is an open access article distributed under the terms of the Creative Commons Attribution License CC-BY 4.0., which permits unrestricted use, distribution, and reproduction in any medium, provided the original author and source are credited.

\section{Case Presentation}

A 70-year-old female with a diagnosis stage II multiple myeloma (MM) by international staging system for multiple myeloma, high risk according to mSMART (Mayo stratification of myeloma and risk-adapted therapy) classification, on chemotherapy with lenalidomide, bortezomib, and dexamethasone presented to our office for a pre-stem cell transplant appointment. In addition to multiple myeloma, she had a past medical history of hyperlipidemia and anxiety. She denied cigarette or alcohol use. She did not have any known cardiovascular history. Family history was significant for myocardial infarction in a brother at the age of 35 and myocardial infarction in her mother at the age of 50. A routine echocardiogram obtained one month prior to stem cell transplant revealed a normal left ventricular ejection fraction of $62 \%$ with a resting tachycardia (Video 1). Two months before she began chemotherapy, her heart rate was $77 \mathrm{bpm}$ on electronic medical records (EMR). She received three cycles of chemotherapy before she presented to the office.

\section{VIDEO 1: Four chamber view showing ejection fraction of $62 \%$ and resting tachycardia}

View video here: https://vimeo.com/436988131

She was admitted to the hospital for an autologous stem cell transplant. Her vitals were significant for a heart rate (HR) of $124 \mathrm{bpm}$, temperature (T) 37 C, respiratory rate (R) 16/min, and blood pressure (BP) 


\section{Cureus}

120/69 mmHg. Baseline electrocardiogram (EKG) at the time of admission showed sinus tachycardia with a heart rate of $113 \mathrm{bpm}$ without any ST-T abnormalities (Figure 1). Her physical examination was significant for tachycardia, regular rhythm, and a grade II/VI systolic murmur in the mitral region. The respiratory examination was clear to auscultation bilaterally. No peripheral edema or jugular venous distension was noted.

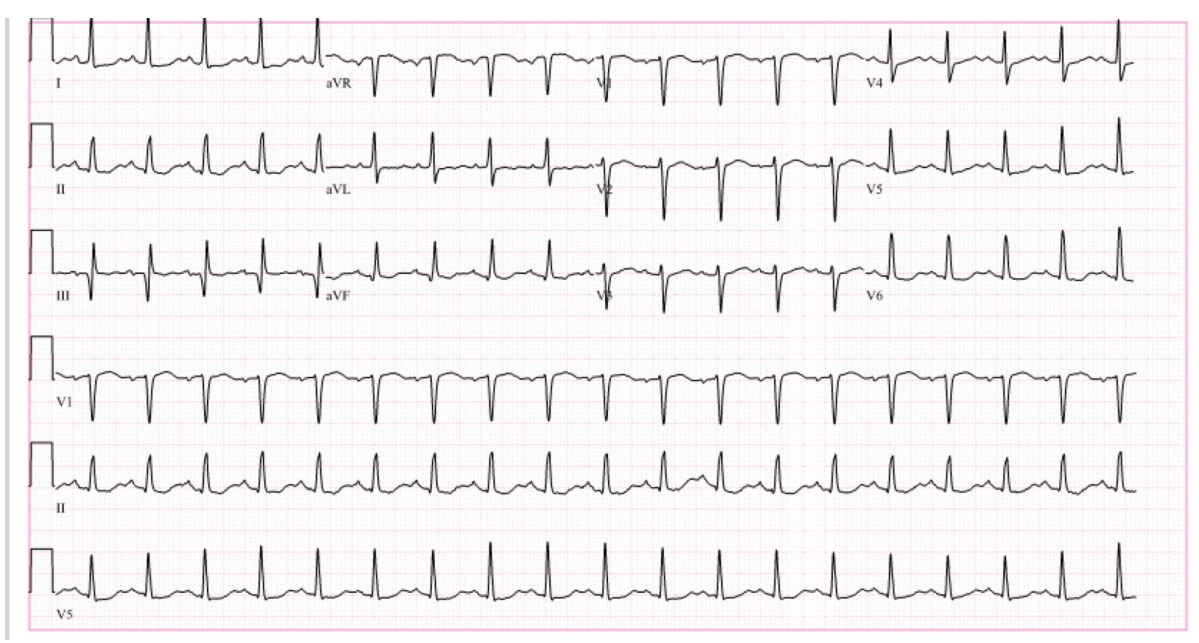

\section{FIGURE 1: Sinus tachycardia with heart rate of 113 beats per minute and non-specific ST-T wave abnormalities}

She was conditioned with melphalan, followed by a successful autologous stem cell transplant. She remained persistently tachycardic despite IV fluids. Sixteen days after stem cell transplant, bibasilar crackles were found via physical exam. Peripheral edema and jugular venous distension were noted. She did not have orthopnea, paroxysmal nocturnal dyspnea, or shortness of breath on exertion.

N-terminal pro b-type natriuretic peptide (NT-proBNP) obtained at the time was elevated at $12,436 \mathrm{pg} / \mathrm{ml}$. A chest X-ray showed pulmonary edema and small bilateral pleural effusions (Figure 2). Electrocardiogram revealed sinus tachycardia with HR of $109 \mathrm{bpm}$ and no evidence of ischemia (Figure 3). A repeat echocardiogram showed worsening systolic function with a calculated ejection fraction of $24.2 \%$ and diffuse hypokinesis of all walls (Video 2). Angiography was not obtained due to suspicion of chemotherapy-induced cardiomyopathy as she had global hypokinesis without segmental wall motion abnormalities. 


\section{Cureus}

(

FIGURE 2: Chest X-ray showing pulmonary vascular congestion and bilateral small pleural effusions

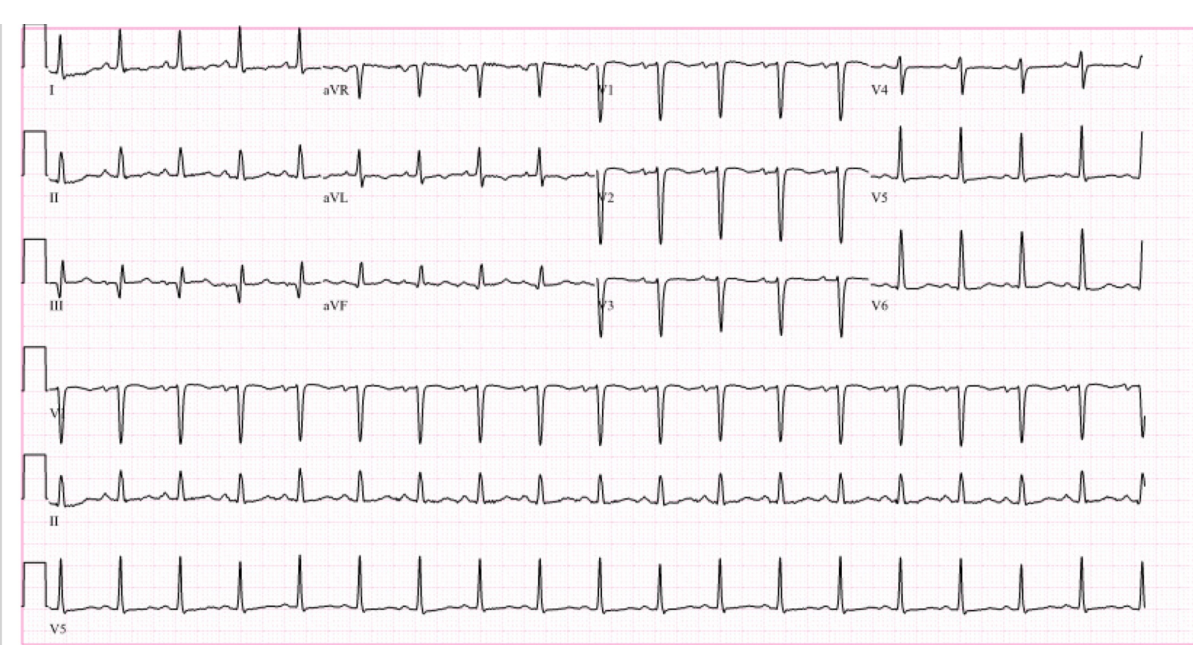

FIGURE 3: Electrocardiogram (EKG) showing sinus tachycardia with heart rate of 109 beats per minute

VIDEO 2: Four chamber view showing calculated ejection fraction of $\mathbf{2 4 . 2} \%$ and diffuse hypokinesis of all walls

View video here: https://vimeo.com/436988516

The patient was initiated on metoprolol tartrate $25 \mathrm{mg}$ twice a day, ivabradine $5 \mathrm{mg}$ twice a day, and losartan $25 \mathrm{mg}$ daily to control tachycardia and medical management of systolic heart failure with reduced ejection fraction. IV furosemide was given for pulmonary congestion while hospitalized. She was discharged on 
metoprolol succinate $200 \mathrm{mg}$ daily, ivabradine $5 \mathrm{mg}$ twice a day, losartan $25 \mathrm{mg}$ daily, and furosemide $20 \mathrm{mg}$ daily.

Two months later, during her follow-up visits, her heart rate remained between $70-80 \mathrm{bpm}$. A repeat echocardiogram performed four weeks later showed partial resolution of heart failure with a visually estimated ejection fraction of $45-50 \%$.

\section{Discussion}

Heart failure in this patient is believed to be due to bortezomib and exacerbated by melphalan. Sinus tachycardia and heart failure were attributed to the use of these agents since she had no sinus tachycardia before starting chemotherapy. This case demonstrates the rare adverse effects of bortezomib and melphalan when used concurrently.

Bortezomib is a dipeptidyl boronic acid and has a role in worsening ischemic heart disease $[14,15]$. It is a potent and reversible inhibitor of $26 \mathrm{~S}$ proteasome. Reduced proteasome activity causes increased apoptosis in smooth muscle cells, which can result in plaque instability and can lead to an increased likelihood of plaque rupture and ischemic complications [7]. Furthermore, biological experiments have shown that bortezomib can cause mitochondrial abnormalities in the cardiac myocytes leading to a decrease in adenosine triphosphate (ATP) synthesis and reduced contractility [13]. Thereby, explaining the underlying etiology of heart failure with the use of bortezomib. However, the effect of bortezomib on cardiac myocytes is reversible since there is no associated apoptosis.

Melphalan cardiotoxicity presents more commonly as atrial fibrillation and supraventricular tachycardia [16], but ventricular arrhythmias have also been reported [17]. There is also an association between melphalan and heart failure [12, 18, 19], but the underlying mechanism is unknown. A cumulative dose of bortezomib is an important consideration because it has been associated with an increased risk of cardiac side effects [7]. Moreover, in experiments with rats, the concurrent use of melphalan along with higher concentrations of bortezomib has been found to increase the cytotoxic effects of melphalan on cardiac myocytes [13]. Our case thus demonstrates the increased risk of heart failure and other cardiac toxicities with the concomitant use of bortezomib and melphalan.

\section{Conclusions}

Cardiomyopathy in our patient was likely a result of the concurrent use of bortezomib and melphalan. Improvement of ejection fraction within four weeks of completion of treatment and starting medical management of heart failure shows reversibility. Sinus tachycardia, with the commencement of bortezomib, has not been observed in prior studies. We believe that even subtle signs such as sinus tachycardia should be given importance for early identification of cardiotoxicity. Thus, we recommend close monitoring of cardiac parameters in patients treated with bortezomib and melphalan.

\section{Additional Information \\ Disclosures}

Human subjects: Consent was obtained by all participants in this study. Conflicts of interest: In compliance with the ICMJE uniform disclosure form, all authors declare the following: Payment/services info: All authors have declared that no financial support was received from any organization for the submitted work. Financial relationships: All authors have declared that they have no financial relationships at present or within the previous three years with any organizations that might have an interest in the submitted work. Other relationships: All authors have declared that there are no other relationships or activities that could appear to have influenced the submitted work.

\section{References}

1. Kazandjian D: Multiple myeloma epidemiology and survival: a unique malignancy . Semin Oncol. 2016, 43:676-681. 10.1053/j.seminoncol.2016.11.004

2. Shaikh AY, Shih JA: Chemotherapy-induced cardiotoxicity. Curr Heart Fail Rep. 2012, 9:117-127. 10.1007/s11897-012-0083-y

3. Tan TC, Neilan TG, Francis S, Plana JC, Scherrer-Crosbie M: Anthracycline-induced cardiomyopathy in adults. Compr Physiol. 2015, 5:1517-1540. 10.1002/cphy.c140059

4. Gurram MK, Pulivarthi S, Ehresmann K, Mathew J: Cardiotoxicity associated with bortezomib: a singlecenter experience. J Cancer Res Ther. 2017, 13:961-963. 10.4103/0973-1482.172126

5. Wang X: Repeated intermittent administration of a ubiquitous proteasome inhibitor leads to restrictive cardiomyopathy. Eur J Heart Fail. 2013, 15:597-598. 10.1093/eurihf/hft069

6. Diwadkar S, Patel AA, Fradley MG: Bortezomib-induced complete heart block and myocardial scar: the potential role of cardiac biomarkers in monitoring cardiotoxicity. Case Rep Cardiol. 2016, 2016:3456287. $10.1155 / 2016 / 3456287$

7. Xiao Y, Yin J, Wei J, Shang Z: Incidence and risk of cardiotoxicity associated with bortezomib in the treatment of cancer: a systematic review and meta-analysis. PLoS One. 2014, 9:e87671.

10.1371/journal.pone.0087671 
8. Voortman J, Giaccone G: Severe reversible cardiac failure after bortezomib treatment combined with chemotherapy in a non-small cell lung cancer patient: a case report. BMC Cancer. 2006, 6:129. 10.1186/1471-2407-6-129

9. Jerkins JH, Suciu A, Mazimba S, Calvo A: Bortezomib-induced severe congestive heart failure Cardiol Res. 2010, 1:20-23. 10.4021/cr105e

10. Hari P, Aljitawi OS, Arce-Lara C, et al.: A phase IIb, multicenter, open-label, safety, and efficacy study of high-dose, propylene glycol-free melphalan hydrochloride for injection (EVOMELA) for myeloablative conditioning in multiple myeloma patients undergoing autologous transplantation. Biol Blood Marrow Transplant. 2015, 21:2100-2105. 10.1016/j.bbmt.2015.08.026

11. San Miguel JF, Schlag R, Khuageva NK, et al.: Bortezomib plus melphalan and prednisone for initial treatment of multiple myeloma. N Engl J Med. 2008, 359:906-917. 10.1056/NEJMoa0801479

12. Bleeker JS, Gertz MA, Pellikka PA, et al.: Cardiomyopathy following high dose melphalan conditioning prior to autologous peripheral blood stem cell transplantation for multiple myeloma and primary amyloidosis. Biol Blood Marrow Transplant. 2011, 17:203. 10.1016/j.bbmt.2010.12.157

13. Nowis D, Mączewski M, Mackiewicz U, et al.: Cardiotoxicity of the anticancer therapeutic agent bortezomib . Am J Pathol. 2010, 176:2658-2668. 10.2353/ajpath.2010.090690

14. Jackson G, Einsele H, Moreau P, San Miguel J: Bortezomib, a novel proteasome inhibitor, in the treatment of hematologic malignancies. Cancer Treat Rev. 2005, 31:591-602. 10.1016/j.ctrv.2005.10.001

15. Versari D, Herrmann J, Gössl M, et al.: Dysregulation of the ubiquitin-proteasome system in human carotid atherosclerosis. Arterioscler Thromb Vasc Biol. 2006, 26:2132-2139. 10.1161/01.ATV.0000232501.08576.73

16. Feliz V, Saiyad S, Ramarao SM, Khan H, Leonelli F, Guglin M: Melphalan-induced supraventricular tachycardia: incidence and risk factors. Clin Cardiol. 2011, 34:356-359. 10.1002/clc.20904

17. Yanamandra U, Gupta S, Khadwal A, Malhotra P: Melphalan-induced cardiotoxicity: ventricular arrhythmias. BMJ Case Rep. 2016, 2016:bcr2016218652. 10.1136/bcr-2016-218652

18. Ritchie DS, Seymour JF, Roberts AW, Szer J, Grigg AP: Acute left ventricular failure following melphalan and fludarabine conditioning. Bone Marrow Transplant. 2001, 28:101-103. 10.1038/sj.bmt.1703098

19. Newbery G, de Alcantara Lima N, Driscoll R, Gurgel LA, Lima CCV: Persistent heart failure following melphalan and fludarabine conditioning. J Cardiol Cases. 2019, 20:88-91. 10.1016/j.jccase.2019.05.001 